\title{
Monotone twist mappings and the calculus of variations
}

\author{
JÜRGEN MOSER \\ Forschungsinstitut für Mathematik, ETH-Zentrum, CH-8092 Zürich, Switzerland
}

(Received 26 June 1985 and revised 4 December 1985)

Abstract. It is shown that a smooth area-preserving monotone twist mapping $\varphi$ of an annulus $A$ can be interpolated by a flow $\varphi^{t}$ which is generated by a $t$-dependent Hamiltonian in $\mathbb{R} \times A$ having the period 1 in $t$ and satisfying a Legendre condition. In other words, any such monotone twist mapping can be viewed as a section mapping for the extremals of variational problem on a torus:

$$
\int F(t, x, \dot{x}) d t,
$$

where $F$ has period 1 in $t$ and $x$ and satisfies the Legendre condition $F_{\dot{x} \dot{x}}>0$.

\section{Introduction}

In a series of papers several authors studied area preserving annulus mappings possessing a certain 'monotone twist' property (see [1], [2], [3]). J. Mather and S. Aubry developed interesting theories for these mappings establishing closed invariant sets for prescribed rotation number, for which this monotone twist property is fundamental. It is the aim of this note to show the close connection of this property and the Legendre condition of calculus of variations. As a matter of fact, the theories of Mather and Aubry can readily be extended to variational problems on the torus satisfying the Legendre condition. Instead of developing such a theory we want to show here by elementary arguments that every monotone twist mapping of an annulus can be viewed as the 'time-one' map of a Hamiltonian system whose Hamiltonian is periodic in $t$ and satisfies the Legendre condition. In other words, every monotone twist mapping can be viewed as the Poincare mapping of a flow given by the solutions of Euler equations of a variational problem on a torus satisfying the Legendre condition.

We describe the result more precisely. We consider a $C^{\infty}$-diffeomorphism of an annulus on its covering space in $\mathbb{R}^{2}$

$$
A=\left\{x, y \mid a^{\prime} \leq y \leq a^{\prime \prime}\right\},
$$

the annulus being obtained by identifying points $(x, y),\left(x^{\prime}, y\right)$ for which $x^{\prime}-x$ is an integer. The lifted diffeomorphism $\varphi:(x, y) \rightarrow\left(x_{1}, y_{1}\right)$ is given by

$$
x_{1}=f(x, y), \quad y_{1}=g(x, y) \text {, }
$$

where $f, g \in C^{\infty}(A)$ and

(i) $\partial(f, g) / \partial(x, y) \equiv 1$;

(ii) $f(x+1, y)=f(x, y)+1, g(x+1, y)=g(x, y)$;

(iii) $g(x, y)-y=0$ for $y=a^{\prime}, a^{\prime \prime}$. 
We observe that such mappings are exact symplectic, i.e. satisfy

$$
\oint\left(y_{1} d x_{1}-y d x\right)=0
$$

for any curve $y=u(x), u(x+1)=u(x)+1$ and its image curve. Indeed, this holds clearly for the boundary curve and by the divergence theorem and (i), for any other curve.

Such a diffeomorphism $\varphi$ is called a monotone twist mapping if $\partial f / \partial y \neq 0$ and, without loss of generality, we will assume

(iv) $\partial f / \partial y>0$.

Geometrically, this means that any vertical tangent vector $(0, \lambda), \lambda>0$, is mapped into a vector in the first or fourth quadrant. For the annulus mapping this is viewed as an increasing twist to the right as the 'radius' $y$ increases. Our main result is contained in:

THEOREM 1. Given a $C^{\infty}$-monotone twist mapping $\varphi$ with the above properties there exists a Hamiltonian function $H=H(t, x, y) \in C^{\infty}(\mathbb{R} \times \mathbb{R} \times A)$ with

(a) $H(t+1, x, y)=H(t, x, y)=H(t, x+1, y)$;

(b) $H_{x}(t, x, y)=0$ for $y=a^{\prime}, a^{\prime \prime}$;

(c) $H_{y y}>0$;

and such that the mapping $\varphi_{t}$ taking the initial values $\left(x_{0}, y_{0}\right)$ of the solutions of

$$
\dot{x}=H_{y}(t, x, y), \quad \dot{y}=-H_{x}(t, x, y)
$$

into the values $(x(t), y(t))$ agrees with the given mapping $\varphi$ for $t=1$.

The condition (b) expresses that the boundaries $y=a^{\prime}, a^{\prime \prime}$ are invariant under the flow and (c) expresses the Legendre condition. Indeed, if one introduces $p$ in the standard way by the Legendre transformation

$$
p=H_{y}(t, x, y)
$$

then, for fixed $t, x$, the variable $p$ will vary monotonically in a closed interval $\left[p^{\prime}(t, x), p^{\prime \prime}(t, x)\right]$ as $y$ varies in $\left[a^{\prime}, a^{\prime \prime}\right]$. With the Lagrange function $F(t, x, p)$ defined by

$$
F(t, x, p)=y p-H(t, x, y)
$$

the Hamiltonian differential equation becomes the Euler equation of the variational problem

$$
\int_{t_{1}}^{t_{1}} F(t, x(t), \dot{x}(t)) d t,
$$

and $H_{y y}>0$ becomes the standard Legendre condition

$$
F_{p p}(t, x, p)>0 \text {. }
$$

The converse of the above theorem is, of course, not true; that is, the time-one map of such a Hamiltonian system, or the corresponding Euler equation, need not be a monotone twist mapping, although it has all the other properties (i)-(iii). One has to require in addition that the solutions $x(t)$ of the Euler equation do not have two conjugate points in the interval $[0,1]$. 
To understand the significance of the above interpolation theorem we note that the composition $\varphi_{1} \circ \varphi_{2}$ of two monotone twist mappings need not be a monotone twist mapping. One just has to consider such a monotone twist mapping $\varphi$ with an elliptic fixed point, for which the linearized mapping is a rotation about an angle $\psi$ between $\pi / 2$ and $\pi$. Then for $\varphi^{2}$ the corresponding angle $2 \psi$ lies between $\pi$ and $2 \pi$, hence the vertical $(0,1)$ is mapped into the 2 nd or 3 rd quadrant, and is not a monotone twist mapping. Now it turns out that the theory of Aubry as well as that of Mather can readily be generalized to mappings $\varphi$ which can be represented as composition $\varphi_{1} \circ \varphi_{2} \circ \cdots \circ \varphi_{N}$ of monotone twist mappings and, more generally, to mappings $\varphi$ which can be interpolated by area-preserving mapping $\varphi_{t}$ with $\varphi_{0}=\mathrm{id}$, $\varphi_{1}=\varphi$ such that $\varphi_{t+\varepsilon} \circ \varphi_{t}^{-1}$ is a monotone twist mapping, for sufficiently small $\varepsilon$. The vector field defined by

$$
X_{t}=\frac{d \varphi_{t}}{d t}\left(\varphi_{t}^{-1}\right)
$$

is Hamiltonian and can be written in the form

$$
\dot{x}=H_{y}, \quad \dot{y}=H_{x} .
$$

Hence the mapping $\varphi_{t+\varepsilon} \circ \varphi_{t}^{-1}$ is given by

$$
\begin{aligned}
& x(t+\varepsilon)=x(t)+\varepsilon H_{y}+O\left(\varepsilon^{2}\right) \\
& y(t+\varepsilon)=y(t)-\varepsilon H_{x}+O\left(\varepsilon^{2}\right),
\end{aligned}
$$

and for $\varepsilon>0$ small enough this is a monotone twist mapping if $H_{y y}>0$. Thus the above mentioned theories can be extended to Hamiltonian systems with the condition $H_{y y}>0$ which is equivalent to the Legendre condition. The theory deals with 'minimal solutions' $x(t)$ which are characterized by the condition that

$$
\int_{-\infty}^{+\infty}(F(t, x+\xi, \dot{x}+\dot{\xi})-F(t, x, \dot{x})) d t \geq 0
$$

for all $\xi \in C_{\text {comp }}^{1}(\mathbb{R})$. Our theorem shows that such a theory is a genuine generalization of the theory of Aubry since every monotone twist mapping can be obtained from such a variational problem, at least for $C^{\infty}$-mappings (see also [4]).

It is rather straightforward to develop such a theory for minimal solutions for variational problems (1.4) satisfying (1.5) which we will describe elsewhere. Here it is our aim to relate the monotone twist condition (iv) to the Legendre condition. Since such variational problems occur quite frequently in mechanics and geometry, it also seems more natural to work with the continuous problem instead of the discrete system. Indeed, as a rule such mappings $\varphi$ are obtained from the continuous problem as section mappings.

Actually such a theorem could be generalized to $C^{r}$-mappings and to mappings in half- or full cylinders with various asymptotic restrictions. But we forgo such extensions and present the proof of the above theorem in the following sections.

I would like to express my thanks to D. Salamon for pointing out inaccuracies and for proof-reading.

\section{Extension of the mapping to $\mathbb{R}^{2}$}

For technical reasons it is useful to extend the mapping $\varphi$ from $A$ to $\mathbb{R}^{2}$ preserving the above mentioned properties (i)-(iv). For this purpose we note that $g_{x}=0$ for 
$y=a^{\prime}, a^{\prime \prime}$ and hence

$$
1=f_{x} g_{y},
$$

i.e. $f_{x} \neq 0$, and because of (ii) $f_{x}>0$. In a sufficiently small strip $a^{\prime \prime}-\varepsilon<y \leq a^{\prime \prime}, f_{x}>0$ and thus $\varphi$ can be represented with a generating function $x_{1} y+w\left(x_{1}, y\right)$ as

$$
\begin{gathered}
x=x_{1}+w_{y}\left(x_{1}, y\right), \\
y_{1}=y+w_{x_{1}}\left(x_{1}, y\right),
\end{gathered}
$$

where $w \in C^{\infty}$ has period 1 in $x_{1}$, satisfies $1+w_{x_{1} y}>0$ and $w_{x_{1}}=0$ for $y=a^{\prime \prime}$. The condition (iv) becomes

$$
w_{y y}<0 \text {. }
$$

The plan is to extend $w\left(x_{1}, y\right)$ for $y \geq a^{\prime \prime}$ as a $C^{\infty}$-function. We use a standard device of $\mathrm{E}$. Borel. For simplicity of the notation we replace $a^{\prime \prime}$ by 0 by a translation and denote the Taylor expansion of $w\left(x_{1}, y\right)$ for $y<0=a^{\prime \prime}$ by

$$
\sum w_{\nu}\left(x_{1}\right) y^{\nu}
$$

We introduce a cut-off function $\zeta \in C^{\infty}(\mathbb{R}), 0 \leq \zeta \leq 1$ with $\zeta(s)=1$ for $s \leq 0, \zeta(s)=0$ for $s \geq 1$. For a given small constant $\rho>0$ we define a sequence

$$
\lambda_{\nu}=2^{\nu} \rho^{-1}\left(1+\max _{x_{1}}\left|w_{\nu}\left(x_{1}\right)\right|\right) \text {, }
$$

set

and define the extension by

$$
\zeta_{\nu}=\zeta\left(\lambda_{\nu} y\right)
$$

$$
\tilde{w}\left(x_{1}, y\right)= \begin{cases}w(x, y) & \text { for } a^{\prime} \leq y \leq 0 \\ \sum_{\nu=0}^{2} w_{\nu}\left(x_{1}\right) y^{\nu}+R\left(x_{1}, y\right) & \text { for } y>0\end{cases}
$$

with

$$
R\left(x_{1}, y\right)=\sum_{\nu=3}^{\infty} w_{\nu}\left(x_{1}\right) y^{\nu} \zeta_{\nu}(y)
$$

For any fixed $y>0$ this sum is finite as $\lambda_{\nu} y \rightarrow \infty$ and defines a $C^{\infty}$-function for $y>0$. To show that $\tilde{w}$ is $C^{\infty}$ at $y=0$ we verify that the Taylor expansions match from both sides at $y=0$. To check this up to order $N-1$ it suffices to estimate the tail:

since $y \zeta_{\nu} \leq \lambda_{\nu}^{-1}$.

$$
\begin{aligned}
\sum_{\nu \geq N+1}\left|w_{\nu}\left(x_{1}\right) y^{\nu} \zeta_{\nu}(y)\right| & \leq \sum_{\nu \geq N+1}\left|w_{\nu}\right| y^{\nu-1} \cdot \lambda_{\nu}^{-1} \\
& \leq y^{N} \rho \sum_{\nu=N+1}^{\infty} 2^{-\nu}=\rho\left(\frac{y}{2}\right)^{N}
\end{aligned}
$$

Similarly, using that $y \zeta_{\nu}^{\prime}, y^{2} \zeta_{\nu}^{\prime \prime}$ are bounded uniformly in $y, \nu$ one checks readily

$$
|R|,|D R|,\left|D^{2} R\right|=O(\rho)
$$

for $y \geq 0$ where $D$ stands for derivatives with respect to $x_{1}$ and $y$. Thus, if $\rho$ is taken small enough we have $\tilde{w}_{y y}<0$ for all $y>0$, and $1+\tilde{w}_{x_{1} y}>0$ for $0 \leq y \leq \rho$.

Next we replace the term $w_{2}(x) y^{2}$ by $\psi=a(x)+b(x) y-(c / 2) y^{2}$ with a positive constant $c$ by the following construction: For a given $c>0$ we define

$$
\gamma(x, y)=-\frac{c}{2}+\left(w_{2}(x)+\frac{c}{2}\right) \zeta\left(\frac{y}{\rho}\right)<0
$$


for all $y>0$ and set

$$
\psi(x, y)=2 \int_{0}^{y} \int_{0}^{y^{\prime}} \gamma\left(x, y^{\prime \prime}\right) d y^{\prime \prime} d y^{\prime}=2 y^{2} \int_{0}^{1}(1-s) \gamma(x, s y) d s .
$$

Then we have

$$
\begin{aligned}
\psi(x, y) & =w_{2}(x) y^{2} \quad \text { for } y>0 \text { near zero } \\
& =a(x)+b(x) y-(c / 2) y^{2} \quad \text { for } y>\rho . \\
\psi_{y y} & =2 \gamma<0 . \\
\psi_{x y} & =2 w_{2}^{\prime} \rho \int_{0}^{y / \rho} \zeta(\lambda) d \lambda=O(\rho) .
\end{aligned}
$$

Therefore the function

$$
w^{*}\left(x_{1}, y\right)=\left\{\begin{array}{l}
w(x, y) \quad \text { for } a^{\prime} \leq y<0 \\
w_{0}\left(x_{1}\right)+w_{1}\left(x_{1}\right) y+\psi\left(x_{1}, y\right)+R\left(x_{1}, y\right)
\end{array}\right.
$$

satisfies

$$
1+w_{x_{1} y}^{*}=1+w_{1}^{\prime}(x)+O(\rho)>0, \quad w_{y y}^{*}<0
$$

for all $y>0$ and $w^{*}\left(x_{1}, y\right)=w_{0}^{*}\left(x_{1}\right)+w_{1}^{*}\left(x_{1}\right) y-(c / 2) y^{2}$ for $y>\rho$. Therefore the relations

$$
\begin{array}{r}
x=x_{1}+w_{y}^{*}\left(x_{1}, y\right) \\
y_{1}=y+w_{x_{1}}^{*}\left(x_{1}, y\right)
\end{array}
$$

define an area-preserving mapping for $a^{\prime} \leq y<\infty$ extending the given map. By construction this is a monotone twist mapping which commutes with $(x, y) \rightarrow$ $(x+1, y)$.

Finally we can modify the extended mapping such that it preserves the circles $y=r$ for sufficiently large constants $r$. We replace $w^{*}$ for $y>\rho$ by

$$
w^{*}\left(x_{1}, y\right)=w_{0}^{*}\left(x_{1}\right)+w_{1}^{*}\left(x_{1}\right) \xi(y)-\frac{c}{2} y^{2}, \quad \xi(y)=\int_{0}^{y} \zeta\left(\varepsilon\left(y^{\prime}-\rho\right)\right) d y^{\prime} .
$$

With a small constant $\sigma=\varepsilon^{2}$ we define

$$
\hat{w}\left(x_{1}, y\right)=w^{*}\left(x_{1}, y\right)+(\zeta(\sigma(y-\rho))-1)\left(w^{*}\left(x_{1}, y\right)+\frac{c}{2} y^{2}\right)
$$

so that $\hat{w}=w$ for $a^{\prime} \leq y<0$, and $\hat{w}=-(c / 2) y^{2}$ for $y>\rho+\sigma^{-1}$. Moreover,

$$
\begin{aligned}
1+\hat{w}_{x_{1} y} & =1+\zeta w_{x_{1} y}^{*}+O(\varepsilon)=1+w_{1}^{\prime}(x) \zeta \xi^{\prime}+O(\rho)+O(\varepsilon)>0 \\
\hat{w}_{y y} & =\zeta w_{y y}^{*}+(\zeta-1) c+O(\sigma)<0
\end{aligned}
$$

for all $y>0$ if $\rho, \sigma=\varepsilon^{2}$ are sufficiently small.

Since $\hat{w}\left(x_{1}, y\right)=-(c / 2) y^{2}$, for $y>\rho+\sigma^{-1}$ the extended mapping has the form $x_{1}=x+c y, y_{1}=y$ for $y>\rho+\sigma^{-1}$.

Similarly, one can extend the mapping $\varphi$ to $y<a^{\prime}$ and thus to $\mathbb{R}^{2}$ preserving all the properties. In addition we have $g \equiv y$ and $f=x+c y$ for large $|y|$.

In the following we consider $C^{\infty}$-monotone twist mappings $\varphi$ in the whole plane with the additional assumption that for each fixed $x$ the monotone function $f(x, \cdot)$ takes on all values, i.e. $f(x,+\infty)=+\infty, f(x,-\infty)=-\infty$. Actually, we impose the 
more stringent condition that with a fixed constant $\delta>0$

$$
\delta \leq f_{y}(x, y) \leq \delta^{-1},
$$

for all $x, y \in \mathbb{R}^{2}$. For the extension constructed above this requirement is clearly satisfied.

We represent $\varphi$ in terms of a generating function $h=h\left(x, x_{1}\right) \in C^{\infty}\left(\mathbb{R}^{2}\right)$ : Solving $x_{1}=f(x, y)$ for $y$ we can write $\varphi$ in the form

$$
y=-h_{x}\left(x, x_{1}\right), \quad y_{1}=h_{x_{1}}\left(x, x_{1}\right)
$$

where $-h_{x}(x, \cdot)$ is the inverse function of $f(x, \cdot)$ hence also monotone and surjective. The assumption $(2.1)$ is equivalent to

$$
\delta \leq-h_{x x_{1}} \leq \delta^{-1} \text {. }
$$

Moreover, the periodicity condition (ii) becomes

$$
h\left(x+1, x_{1}+1\right)=h\left(x, x_{1}\right) .
$$

First we prove the analogue of theorem 1 for such mappings in the plane.

THEOREM 2. Let $\varphi$ be a monotone twist mapping of $\mathbb{R}^{2}$ into itself given by a generating function $h \in C^{\infty}\left(\mathbb{R}^{2}\right)$, satisfying (2.3) and (2.4). Then there exists Hamiltonian function $H=H(t, x, y) \in C^{\infty}\left([0,1] \times \mathbb{R}^{2}\right)$ with the following properties

(a) $H(t, x, y)=H(t, x+1, y)$;

(b) $0<\delta \leq H_{y y} \leq \delta^{-1}$;

and the mapping $\varphi_{t}$ taking initial values $\left(x_{0}, y_{0}\right)$ of the solutions of

$$
\dot{x}=H_{y}, \quad \dot{y}=-H_{x}
$$

into $(x(t), y(t))$ agrees with the given mapping $\varphi$ for $t=1$.

In the next section we shall prove this theorem and subsequently derive theorem 1 from it by smoothing the flow obtained by periodic extension with respect to $t$ and finally, by taking care of the boundary condition (b) in theorem 1.

\section{Proof of theorem 2}

The proof will be based on the construction of a variational principle (1.3) for which the extremal integral

$$
S\left(x_{0}, x_{1}\right)=\int_{0}^{1} F(t, x(t), \dot{x}(t)) d t
$$

taken along extremals $x(t)$ connecting $x_{0}=x(0)$ and $x_{1}=x(1)$ agrees with $h\left(x_{0}, x_{1}\right)$. This would imply that the corresponding Hamiltonian flow $\varphi_{t}$ satisfies $\varphi_{1}=\varphi$. Of course, such an integrand is rather arbitrary and we want to require that the extremals are straight lines

$$
x(t)=x_{0}+t\left(x_{1}-x_{0}\right) .
$$

Let us determine the most general such variational principle. From the Euler equations one sees that a necessary and sufficient condition for straight line extremals is that $F$ satisfies the partial differential equation

$$
\left(\partial_{t}+p \partial_{x}\right) F_{p}=F_{x} \text {. }
$$


Differentiation with respect to $p$ gives

$$
\left(\partial_{t}+p \partial_{x}\right) F_{p p}=0
$$

i.e.

$$
F_{p p}(t, x, p)=G(x-p t, p)
$$

for some arbitrary function $G(x, p)$. One verifies readily that the most general solution of (3.3) is given by

$$
\begin{aligned}
F & =F_{0}(t, x, p)+p m_{x}(t, x)+m_{t}(t, x) \\
F_{0} & =\int_{0}^{p}\left(p-p^{\prime}\right) G\left(x-p^{\prime} t, p^{\prime}\right) d p^{\prime} .
\end{aligned}
$$

We determine the corresponding extremal integral (3.1)

$$
S\left(x_{0}, x_{1}\right)=S_{0}\left(x_{0}, x_{1}\right)+m\left(1, x_{1}\right)-m\left(0, x_{0}\right),
$$

where $S_{0}$ is the extremal integral for $F_{0}$. One has the standard relations

$$
S_{x_{0}}=-F_{p}\left(0, x_{0}, x_{1}-x_{0}\right), \quad S_{x_{1}}=F_{p}\left(1, x_{1}, x_{1}-x_{0}\right), \quad S_{x_{0} x_{1}}=-F_{p p}\left(0, x_{0}, x_{1}-x_{0}\right) .
$$

In order to achieve $S=h$ we have to set

$$
G(x, p)=-h_{x_{0} x_{1}}(x, x+p)>0
$$

so that

$$
F_{0}(t, x, p)=-\int_{0}^{p}\left(p-p^{\prime}\right) h_{x_{0} x_{1}}\left(x-p^{\prime} t, x+p^{\prime}(1-t)\right) d p^{\prime} .
$$

Then we have

$$
\frac{\partial^{2} S_{0}}{\partial x_{0} \partial x_{1}}=\frac{\partial^{2} h}{\partial x_{0} \partial x_{1}}
$$

or

$$
S_{0}-h=u\left(x_{0}\right)+v\left(x_{1}\right) .
$$

Finally, if we choose the boundary values of $m(t, x)$ so that

$$
m\left(0, x_{0}\right)=u\left(x_{0}\right) ; \quad m\left(1, x_{1}\right)=-v\left(x_{1}\right)
$$

we obtain from (3.6) and (3.8)

$$
S=h .
$$

We observe that on account of (3.4)

$$
F_{p p}(t, x, p)=-h_{x_{0} x_{1}}(x-p t, x+p(1-t))>0
$$

which shows that the monotone twist condition corresponds to the Legendre condition $F_{p p}>0$. The periodicity condition (2.4) for $h$ yields

$$
F_{0}(t, x+1, p)=F_{0}(t, x, p)
$$

and if we choose, for example

$$
m(t, x)=(1-t) u(x)-t v(x), \quad 0 \leq t \leq 1
$$

one computes readily

$$
m_{x}=-(1-t) h_{x_{0}}(x, x)+t h_{x_{1}}(x, x), \quad m_{t}=h(x, x) .
$$

so that $m_{x}, m_{t}$ and hence $F(t, x, p)$ have period 1 in $x$. 
Thus the variational principle is constructed with the desired properties and the Hamiltonian $H(t, x, y)$ is obtained by the Legendre transformation

$$
y=F_{p}(t, x, p) ; \quad H(t, x, y)=y p-F(t, x, p) .
$$

Because of (3.9) we have

$$
0<\delta \leq F_{p p} \leq \delta^{-1}
$$

and because of $H_{y y} \cdot F_{p p}=1$ also

$$
0<\delta \leq H_{y y} \leq \delta^{-1} \text {. }
$$

Moreover, $H$ has period 1 in $x$. From standard facts of calculus of variations it follows that the flow $\varphi_{t}$ of the Hamiltonian system for $H$ interpolates $\varphi$ and the identity, proving theorem 2 .

We illustrate this construction with a mapping

$$
\varphi(x, y)=\left(x+y+V_{x}(x), y+V_{x}(x)\right)
$$

with the generating function

$$
h\left(x, x_{1}\right)=\frac{1}{2}\left(x_{1}-x\right)^{2}+V(x)
$$

where $V$ is smooth function of period 1. The formulae (3.7), (3.10) give for $0 \leq t<1$

$$
F(t, x, p)=\frac{1}{2} p^{2}-(1-t) p V_{x}+V
$$

and the Hamiltonian

$$
H(t, x, y)=\frac{1}{2}\left(y+(1-t) V_{x}\right)^{2}-V .
$$

Periodic continuation gives rise to discontinuities. A more suggestive yet more singular choice is

$$
F(t, x, p)=\frac{1}{2} p^{2}+\delta_{\text {per }}(t) V(x)
$$

where $\delta_{\text {per }}$ is the periodic Dirac function with support on the integers.

\section{Smoothing}

In order to obtain a variational problem periodic of period 1 in $t$ we just have to extend $F(t, x, y)$, or equivalently $H(t, x, y)$, defined in $t \in[0,1)$ periodically, by setting $F(t, x, y)=F(t-j, x, y)$ with an integer $j \in[t, t+1)$. This is sufficient for most purposes though it leads to discontinuities of $F, H$ and the corresponding vector field at integer values of $t$, while the corresponding flow is continuous. However, in this section we want to show that $F, H$ can in fact be replaced by smooth periodic functions $\tilde{F}, \tilde{H}$ which give rise to the same given Poincaré mapping and satisfy the Legendre condition. To achieve this we will replace $\varphi_{t}$ by another interpolating flow $\tilde{\varphi}_{t}$ which agrees with $\varphi_{t}$ for $t=0,1$ and which corresponds to a smooth $t$-periodic Hamiltonian $\tilde{H}$ satisfying $\tilde{H}_{y y}>0$. This will be done with the following somewhat clumsy argument.

For simplicity we restrict ourselves in this section to monotone twist mappings satisfying

$$
f(x, y)=x+c y, \quad g(x, y)=y \quad \text { for }|y| \text { large. }
$$

This condition holds for the extension of the annulus mapping constructed in $\S 2$, and since we now turn to the proof of theorem 1 we may as well assume (4.1) to 
simplify the following. By (2.2) this assumption is equivalent to

$$
h=\frac{1}{2 c}\left(x_{1}-x\right)^{2}+\text { const. for large }\left|x_{1}-x\right|
$$

where the constant for $\pm\left(x_{1}-x\right)$ large need not be the same. Using the formulae (3.7), (3.10) defining $F$ we find

$$
F=\frac{1}{2 c} p^{2}+\text { const. for }|p| \text { large }
$$

or

$$
H=\frac{c}{2} y^{2}+\text { const. for }|y| \text { large. }
$$

We define the exact symplectic flow

$$
\psi^{s}:(x, y) \rightarrow(x+s y, y)
$$

corresponding to the Hamiltonian $\frac{1}{2} y^{2}$. For $\varepsilon \in\left(0, \frac{1}{2}\right)$ we consider

$$
\varphi_{1}=\psi^{-\varepsilon} \circ \varphi \circ \psi^{-\varepsilon}
$$

which for $\varepsilon$ sufficiently small is a monotone twist mapping. Applying theorem 2 to $\varphi_{1}$ we obtain an interpolating flow $\varphi_{\tau}, 0 \leq \tau \leq 1$ with $\varphi_{\tau}=\mathrm{id}, \varphi_{1}$ for $\tau=0,1$, respectively. Now we define

$$
\chi_{\mathrm{t}}= \begin{cases}\psi^{t} & \text { for } 0 \leq t<\varepsilon \\ \varphi_{\tau} \circ \psi^{\varepsilon} & \text { for } \tau=(t-\varepsilon) /(1-2 \varepsilon), \quad \varepsilon \leq t \leq 1-\varepsilon \\ \psi^{t-1} \circ \varphi & \text { for } 1-\varepsilon<t<1\end{cases}
$$

which is continuous for $t \in[0,1)$ and interpolates $\chi_{0}=\mathrm{id}$ and $\chi_{1}=\varphi$. Moreover, $\chi_{t}$ is smooth except for $t=\varepsilon, 1-\varepsilon$. This flow $\chi_{t}$ is generated by the piecewise smooth Hamiltonian

$$
\hat{H}= \begin{cases}\frac{1}{2} y^{2} & \text { for } t \in[0, \varepsilon) \text { and } t \in(1-\varepsilon, 1) \\ \frac{1}{1-2 \varepsilon} H(\tau, x, y) & \text { with } \tau=(t-\varepsilon) / 1-2 \varepsilon, t \in[\varepsilon, 1-\varepsilon]\end{cases}
$$

For large $|y|$ we have

$$
\hat{H}=\frac{1}{2} y^{2}+\text { const. }
$$

if we choose the constant $c=1-2 \varepsilon$ in $\S 2$, so that $\hat{H}_{x}, \hat{H}_{y}-y$ have compact support. Moreover, we have $\hat{H}_{y y}>0$.

Since $\hat{H}=\hat{H}(t, x, y)$ agrees for $t$ near 0 and 1 with $\frac{1}{2} y^{2}$ the periodic extension of $\hat{H}$ does not lead to discontinuities at integers $t$; instead we have discontinuities of $\hat{H}$ for $t=\varepsilon, 1-\varepsilon(\bmod 1)$ which we will now remove, by smoothing $\chi_{t}$ near $t=\varepsilon$, $1-\varepsilon$ without changing it near the endpoints. This is actually a local problem.

Before proceeding we point out a difficulty. One may try to achieve the desired smoothing by representing $\chi_{t}$ in terms of a generating function, say $x y_{1}+\hat{w}_{t}\left(x, y_{1}\right)$ and replace $\hat{w}_{1}$ by a smooth $\tilde{w}_{t}$ which for fixed $t$ is $C^{2}$-close to $\hat{w}_{t}$ and agrees with $\hat{w}_{t}$ outside an interval about $\varepsilon$. This would give rise to a smooth symplectic flow $\tilde{\varphi}_{t}$, $C^{3}$-close to $\chi_{t}$, and the Hamiltonian $\tilde{H}(t, x, y)$ generating $\tilde{\varphi}$, would be smooth at 
$\varepsilon$. However, the difficulty is to verify the Legendre condition near $t=\varepsilon$, since $\tilde{H}_{y y}$ cannot be uniformly close to $\hat{H}_{y y}$ since the latter is discontinuous.

To avoid this difficulty we proceed differently and first approximate $\hat{H}$ by a smooth $H^{*}$ such that $H_{y y}^{*}>0$. Secondly we will change the corresponding flow $\varphi_{t}^{*}$ outside an interval about $t=\varepsilon$ to a flow $\tilde{\varphi}_{t}$ agreeing with $\varphi_{t}$ outside. With appropriate estimates we can ensure that the Hamiltonian $\tilde{H}$ generating $\tilde{\varphi}_{t}$ satisfies the Legendre condition.

To first smooth $\hat{H}$ near $t=\varepsilon$ we set for $0<\rho<\varepsilon$

$$
H^{*}(t, x, y)=\hat{H} * \eta_{\rho}, \quad \eta_{\rho}=\rho^{-1} \eta\left(\rho^{-1} t\right)
$$

where $\eta \in C^{\infty}(\mathbb{R}), 0 \leq \eta \leq 1, \eta(s)=0$ for $|s| \geq 1$, and

$$
\int \eta(s) d s=1 \text {, }
$$

so that $H^{*}$ is $C^{\infty}$ for $0 \leq t \leq \frac{1}{2}$ and $H^{*}=H$ for $t<\varepsilon-\rho$. Moreover, since $\eta \geq 0$ we have $H_{y y}^{*}>0$.

Moreover, with $D^{\alpha}=\partial_{x}^{\alpha_{1}} \partial_{y}^{\alpha_{2}}, \alpha=\left(\alpha_{1}, \alpha_{2}\right)$ we find

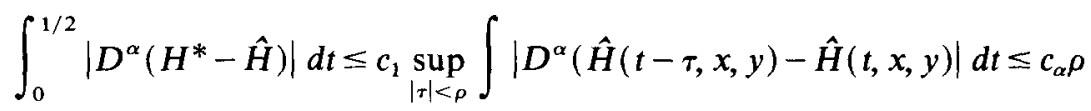

since $D^{\alpha} \hat{H}$ is piecewise smooth in $t$. On the other hand the uniform estimate

$$
\sup _{|t-\varepsilon| \geq \rho}\left|D^{\alpha}\left(H^{*}-\hat{H}\right)\right| \leq c_{2} \sup _{|\tau|<\rho}\left|D^{\alpha}(\hat{H}(t-\tau, x, y)-\hat{H}(t, x, y))\right| \geq c_{\alpha} \rho
$$

holds only for $|t-\varepsilon| \geq \rho$. All these estimates are uniform in $x, y$ since $H^{*}-\hat{H}$ has compact support for $0<t \leq \frac{1}{2}$.

If $\varphi_{t}^{*}$ denotes the flow determined by $H^{*}$ one derives from standard theorems on solutions of differential equations

$$
\left|\varphi_{t}^{*}-\chi_{t}\right|_{m} \leq c_{3} \int_{0}^{\frac{1}{2}}\left|H^{*}-\hat{H}\right|_{m+1} d t=O(\rho)
$$

for $0 \leq t \leq \frac{1}{2}$ where for a function $G=G(t, x, y)$ the norm is defined by

$$
|G|_{m}=\sup _{x, y} \sum_{|\alpha| \leq m}\left|D^{\alpha} G\right| ; \quad|\alpha|=\alpha_{1}+\alpha_{2} .
$$

For the $t$-derivatives one obtains from the differential equation and (4.6)

$$
\left|\partial_{t}\left(\varphi_{t}^{*}-\chi_{t}\right)\right|_{m} \leq c_{4}\left|H^{*}-\hat{H}\right|_{m+1}=O(\rho)
$$

only for $|t-\varepsilon| \geq \rho$. Also $\varphi_{t}^{*}=\chi_{t}$ for $0 \leq t<\varepsilon-\rho$.

It remains to change $\varphi_{t}^{*}$ for $t>t_{2}>\varepsilon$ so that it agrees with $\chi_{t}$ there and still retains all required properties. For this purpose we represent $\chi_{t}, \varphi_{t}^{*}$ with generating functions $x_{1} y+\hat{w}\left(t, x_{1}, y\right)$ and $x_{1} y+w^{*}\left(t, x_{1}, y\right)$ respectively, so that $\hat{w}, w^{*}$ have period 1 in $x_{1}$.

Such a representation is possible for $t \leq 2 \varepsilon, \varepsilon$ sufficiently small, since $\chi_{t}, \varphi_{t}^{*}$ are then close to the identity mapping. On account of $(4.7),(4.8)$ one has

$$
\begin{aligned}
\left|w^{*}-\hat{w}\right|_{m} & =O(\rho) & & \text { for } t \leq 2 \varepsilon \\
\mid \partial_{t}\left(w^{*}-\left.\hat{w}\right|_{m}\right. & =O(\rho) & & \text { for } \varepsilon+\rho \leq t \leq 2 \varepsilon .
\end{aligned}
$$


Moreover,

$$
w^{*}=\hat{w} \quad \text { for } 0<t<\varepsilon-\rho .
$$

With $t_{1}, t_{2}$ in $\varepsilon<t_{1}<t_{2}<2 \varepsilon$ use a cut-off function $\xi(t) \in C^{\infty}, \xi=1$ for $t<t_{1}$ and $\xi=0$ for $t>t_{2}$ and define

$$
\tilde{w}=(1-\xi) \hat{w}+\xi w^{*}
$$

so that $\tilde{w}=w^{*}$ for $t<t_{1}$ hence smooth and $\tilde{w}=\hat{w}$ for $t>t_{2}$. Moreover, $\tilde{w}=\hat{w}$ also for $0<t<\varepsilon-\rho$, hence the flow $\tilde{\varphi}_{t}$ defined by $\tilde{w}$ is smooth in $0 \leq t \leq \frac{1}{2}$ and agrees with $\chi_{\mathrm{r}}$ near the end-points. It remains to verify that the Hamiltonian $\tilde{H}$ corresponding to $\tilde{\varphi}$, satisfies the Legendre condition.

For this purpose we express the Legendre condition in terms of $w$ : If the flow $(x, y) \rightarrow\left(x^{\prime}=x(t), y^{\prime}=y(t)\right)$ is defined by the Hamiltonian $H=H\left(t, x^{\prime}, y^{\prime}\right)$ then one has by standard transformation theory

$$
H\left(t, x^{\prime}, y^{\prime}\right)=-\partial_{t} w\left(t, x^{\prime}, y\right)
$$

from which one finds

$$
H_{y^{\prime}}=\frac{-w_{t y}}{1+w_{x^{\prime} y}} .
$$

Thus the condition $H_{y^{\prime} y^{\prime}}>0$ is equivalent to

$$
w_{t y y}\left(1+w_{x^{\prime} y}\right)-w_{t y} w_{x^{\prime} y y}<0 .
$$

By construction this condition is valid for $w=\hat{w}$ as well as for $w^{*}$, and by continuity for a $C^{3}$-neighbourhood of these. We have to verify this condition for $\tilde{w}$ only in $\left[t_{1}, t_{2}\right]$ where by $(4.9),(4.11)$

$$
|\tilde{w}-\hat{w}|_{3}=O(\rho),
$$

i.e. for $\rho$ sufficiently small $(4.12)$ is satisfied for $w=\tilde{w}$, i.e. $\tilde{H}_{y y}>0$ in $\left(0, \frac{1}{2}\right)$.

This way the discontinuity at $t=\varepsilon$ is removed, and by the same argument one gets rid of the discontinuity at $t=1-\varepsilon$ to obtain a smooth Hamiltonian $\tilde{H}(t, x, y)$ of period 1 in $t$, and $x$ with $\tilde{H}_{y y}>0$ for which the Poincare mapping agrees with the given $\varphi$.

\section{Boundary condition}

Finally we will take care of the boundary condition (b) in theorem 1 . The Hamiltonian $\tilde{H}$ constructed in the previous section will be called $H$; it belongs to $C^{\infty}\left(\mathbb{R}^{3}\right)$ and satisfies conditions (a), (c) of that theorem. The flow $\varphi_{t}$ takes the boundary curves $y=a^{\prime}, a^{\prime \prime}$ into curves $\Gamma_{t}^{\prime}, \Gamma_{t}^{\prime \prime}$ with

$$
\Gamma_{j}^{\prime}=\Gamma_{0}^{\prime}, \quad \Gamma_{j}^{\prime \prime}=\Gamma_{0}^{\prime \prime} \quad \text { for } j=0, \pm 1, \ldots
$$

since by assumption $\varphi$ leaves these curves invariant.

Lemma. The curves $\Gamma_{t}^{\prime}, \Gamma_{t}^{\prime \prime}$ are graphs of $C^{\infty}$-functions

of period 1 in $t, x$.

$$
y=u^{\prime}(t, x) ; \quad y=u^{\prime \prime}(t, x)>u^{\prime}(t, x)
$$

Thus the image of the annulus $A$ under $\varphi_{t}$ is given by

$$
A_{t}: u^{\prime}(t, x) \leq y \leq u^{\prime \prime}(t, x) \text {. }
$$


To prove this lemma we set $y=a$, where $a=a^{\prime}$ or $a^{\prime \prime}$ and consider the curve $\Gamma_{t}$ given by

$$
(x, y)=\varphi_{t}\left(x_{0}, a\right)=\left(x\left(t, x_{0}, a\right), y\left(t, x_{0}, a\right)\right) .
$$

We define the tangent vector

$$
(X, Y)=\frac{\partial \varphi_{t}\left(x_{0}, a\right)}{\partial x_{0}}=d \varphi_{t}(1,0) \neq 0
$$

Thus $X=X\left(t, x_{0}\right), Y=Y\left(t, x_{0}\right)$ have period 1 in $x_{0}$ and $(X, Y)$ describes a closed curve $\gamma_{t}$ for each fixed $t$, depending continuously on $t$. For $t=0$ we have $(X, Y)=$ $(1,0)$ and, more generally,

$$
X>0 \quad \text { for } t=j \in \mathbb{Z} \text { and all } x_{0},
$$

since $y=a$ is invariant under $\varphi^{j}$ and the induced mapping is a diffeomorphism. We want to prove $X>0$ for all $t \geq 0$.

Assume that $t_{0}>0$ is the first zero of $\inf _{x_{0}} X$, so that $X>0$ for $t \in\left(0, t_{0}\right)$ and all $x_{0}$, but for some $x_{0}^{*}$ we have

$$
X\left(t_{0}, x_{0}^{*}\right)=0 .
$$

Then the differential equation $\dot{X}=H_{y x} X+H_{y y} Y$ shows that

$$
\dot{X}\left(t_{0}, x_{0}^{*}\right) \neq 0
$$

hence

$$
0>\dot{X}\left(t_{0}, x_{0}^{*}\right)=H_{y y} Y
$$

i.e.

$$
Y\left(t_{0}, x_{0}^{*}\right)<0 .
$$

Thus the crossing takes place on the negative $Y$-axis.

Next we see that such a crossing of $\gamma_{t}$ of the negative $Y$-axis cannot disappear for $t>t_{0}$, and this clearly contradicts (5.2). If this assertion were false there would exist a 'last' crossing $t=t_{1}<1$ on the negative $Y$-axis - since $\gamma_{t}$ depends continuously on $t$ and $X^{2}+Y^{2}>0$. Thus

$$
\begin{gathered}
X\left(t_{1}, x_{0}^{\prime}\right)=0, \quad Y\left(t_{1}, x_{0}^{\prime}\right)<0 \\
X\left(t, x_{0}\right)>0 \quad \text { for } t \in\left(t_{1}, t_{1}+\varepsilon\right) \text { and all } x_{0} .
\end{gathered}
$$

By the same argument as above we conclude that

$$
Y\left(t_{1}, x_{0}\right)>0 \text {. }
$$

This contradiction shows that

$$
X=\frac{\partial x}{\partial x_{0}}\left(t, x_{0}, a\right)>0 \quad \text { for } t \geq 0 .
$$

Eliminating $x_{0}$ from the relations $x=x\left(t, x_{0}, a\right), y=y\left(t, x_{0}, a\right)$ one obtains the representation $y=u(t, x)$ for the curve as a graph proving the lemma. Clearly $u(t, x+1)=u(t, x)$ since $H$ has period 1 in $x$. Moreover, one finds that $\int_{0}^{1} u(t, x) d x$ is independent of $t$, hence

$$
\int_{0}^{1} u^{\prime}(t, x) d x=a^{\prime}, \quad \int_{0}^{1} u^{\prime \prime}(t, x) d x=a^{\prime \prime} .
$$


We seek an exact symplectic transformation $\tau_{t}$ which takes the curves $\Gamma_{t}^{\prime}, \Gamma_{t}^{\prime \prime}$ into $\Gamma_{0}^{\prime}, \Gamma_{0}^{\prime \prime}$. In order not to destroy the Legendre condition we have to require that $\tau_{t}$ preserves the foliation $x=$ const. The most general such transformation has the form

$$
\tau_{t}(x, y)=\left(A(t, x), A_{x}^{-1}(y-B(t, x))\right)
$$

where

$$
A_{x}>0 ; A-x, B \text { of period } 1 \text { in } x \text {. }
$$

Thus the requirement that $\tau_{t}$ takes $\Gamma_{t}^{\prime}, \Gamma_{t}^{\prime \prime}$ into $\Gamma_{0}^{\prime}, \Gamma_{0}^{\prime \prime}$ leads to the equations

$$
A_{x}^{-1}\left(u^{\prime}-B\right)=a^{\prime}, \quad A_{x}^{-1}\left(u^{\prime \prime}-B\right)=a^{\prime \prime},
$$

which yields

$$
A_{x}=\frac{u^{\prime \prime}-u^{\prime}}{a^{\prime \prime}-a^{\prime}}>0, \quad B=\frac{a^{\prime \prime} u^{\prime}-a^{\prime} u^{\prime \prime}}{a^{\prime \prime}-a^{\prime}} .
$$

This determines $B$ uniquely and $A$ up to an irrelevant function of $t$ which we fix by $A(t, 0)=0$. Because of (5.3) the condition (5.4) holds true, as well as $\tau_{j}=\mathrm{id}$ for all integer $j$.

With the so determined $\tau_{t}$ we define the flow

$$
\bar{\varphi}_{t}=\tau_{t} \circ \varphi_{t}
$$

which preserves the curves $\Gamma_{0}^{\prime}, \Gamma_{0}^{\prime \prime}$, and thus gives rise to a flow in $A: a^{\prime} \leq y \leq a^{\prime \prime}$.

From the construction it is clear that the Legendre condition is maintained. One can see this also by determining the Hamiltonian $\bar{H}$ for $\bar{\varphi}_{t}$ which is given by

$$
\bar{H}\left(t, x^{\prime}, y^{\prime}\right)=H(t, x, y)+W_{t}\left(t, x, y^{\prime}\right)
$$

where $\left(x^{\prime}, y^{\prime}\right)=\tau_{t}(x, y)$ and

$$
W=A(t, x) y^{\prime}+\int_{0}^{x} B(t, \lambda) d \lambda
$$

is the generating function of $\tau_{t}$. Hence

$$
\bar{H}_{y^{\prime} y^{\prime}}=A_{x}^{2} H_{y y}>0 .
$$

Moreover, $\bar{\varphi}_{j}=\varphi_{j}$ for all integers $j$. The Hamiltonian $\bar{H}$ restricted to the annulus $A$ satisfies all conditions of theorem 1 .

\section{REFERENCES}

[1] S. Aubry \& P. Y. Le Daeron. The discrete Frenkel Kontorova model and its applications. Physica 8D, (1983) 381-422.

[2] A. Katok. Some remarks on Birkhoff and Mather twist theorems. Ergod. Th. \& Dynam. Sys. 2, (1982) 185-194.

[3] J. N. Mather. Existence of quasi-periodic orbits for twist homeomorphisms of the annulus. Topology 21, (1982) 457-467.

[4] J. Moser. Recent developments in the theory of Hamitonian systems. To appear in SIAM Reviews 1986. 